ISSN: 2386-3919

ISSN electrónico: 2386-3927

DOI: http://dx.doi.org/10.14201/et2015332151170

\title{
COMPETENCIAS DEL MAESTRO MENTOR DE PRÁCTICAS
}

\author{
Competencies of the practicum mentor
}

\section{Les compétences du tuteur de stage}

\author{
Carmen Megia Cuelliga \\ Departamento de Prácticas. Escuela Universitaria ESCUNI (UCM). Madrid. \\ Correo-e: cmegia@escuni.com
}

Recibido: 23-01-2015; Aceptado: 09-03-2015; Publicado: 30-10-2015

BIBLID [2386-3927 (2015) 33, 2; 151-170]

Ref. Bibl. CARMEN MEGIA CUELligA. Competencias del maestro mentor de prácticas. Enseñanza \& Teaching, 33, 2-2015, 151-170. http://dx.doi.org/10.14201/ et2015332151170

RESUMEN: Este artículo es el resultado de un proceso de investigación centrado en la figura del maestro mentor de prácticas. La finalidad del artículo persigue determinar e identificar las competencias profesionales clave que deben tener asumidas y adquiridas y que contribuyen a la formación de los futuros maestros, cuando éstos realizan el periodo de prácticas de magisterio. Por otra parte, queremos explorar cómo se sienten de preparados y formados los maestros mentores para el ejercicio de su labor de mentor.

Los objetivos específicos se centran en:

- Determinar cuáles son las competencias que deben tener asumidas y adquiridas para formar al futuro maestro.

- Contribuir a la formación de los maestros mentores para el desempeño de sus funciones.

En el estudio se ha utilizado una metodología de carácter cuantitativo. Se ha elaborado un cuestionario con escala tipo Likert que se ha aplicado a los maestros mentores de colegios de toda la Comunidad de Madrid con el fin de recoger la mayor información posible sobre las competencias específicas desempeñadas por los mentores durante el periodo de prácticas de enseñanza de los futuros maestros. Participaron 105 colegios durante el curso 2012-2013. La muestra real participante de 
maestros mentores está constituida por 674. La fiabilidad del cuestionario se obtuvo mediante el alpha de Cronbach con fiabilidad del 0,859, en los bloques: el mentor y el centro escolar, por una parte, y, por otra, el mentor y el desarrollo de competencias específicas docentes del alumno en prácticas. Se utilizó para el análisis de resultados el programa estadístico SPSS 20.0, la prueba de contraste mediante ANOVA y para el análisis de segmentación se utilizó el método de crecimiento CHAID para identificar el nivel de satisfacción de los mentores con respecto a su formación y capacidad para ejercer su función de mentor.

Entre los hallazgos encontrados destacamos la importancia de la relación entre el mentor y el estudiante en prácticas, por un lado, al establecer un clima de confianza y asesoramiento que facilitará su desarrollo profesional, y, por otro, queda constatada la profesionalidad y capacitación que poseen los mentores en el ejercicio de su labor. Como conclusiones y propuestas futuras de formación se evidencia la necesidad de ofrecer a los mentores una formación continua que facilite el desempeño de sus tareas en el acompañamiento del estudiante de magisterio en prácticas.

Palabras clave: mentor; tutor; competencias; prácticum; prácticas; formación.

SUMMARY: This article is the result of research centered on the figure of the primary school teacher practicum mentor. The article aims to determine and identify the main professional competencies that these mentors should assume and acquire and that will contribute to the future primary school teachers training during their teacher-practicum period. Additionally, we wish to explore how prepared the teachers' mentors feel to undertake their role as mentors.

The research specific goals are:

- Determine which competencies the mentors of future primary school teachers should assume and acquire.

- Contribute to the training of the mentors in order enable them to fulfill their roles.

This research uses a quantitative analysis methodology. A questionnaire utilizing the Likert scale has been elaborated and applied to practicum mentors of the Community of Madrid with the purpose of collecting as much information as possible about the specific competencies assumed by the mentors during the practicum period. During the 2012-2013 school year, 105 schools took part in the research. The real sample consists of 674 teachers. The fiability of the questionnaire was obtained by Cronbach's Alpha with a 0,859 fiability in the blocs: the tutor and the teaching institutions on one hand and the tutor and the development of the teacher's specific competencies during the practicum. SPSs 20.0 software has been used to analyze the results and back-testing has been done with ANOva. For segmentation analysis, Chaid's growth method has been used in order to identify the mentor's level of satisfaction with their ability to exercise their role as mentors.

Among the results obtained, we emphasize the importance of the relationship between mentor and student on one hand that will establish an atmosphere of trust and counseling that will facilitate their further professional development. On the other hand, we have corroborated the mentors' abilities and professionalism in the performance of their roles. The conclusions and suggestions focus on the necessity of 
offering mentors continued training that will enhance their performance as mentors and advisors for the future primary school teachers.

Key words: mentor; tutor; competencies; practicum; practicum; training.

RÉSUMÉ: Cet article est le résultat d'un processus de recherche focalisé dans la figure du mentor de stage. Le but de l'article cherche à déterminer et à identifier les compétences professionnelles clés qui doivent être acquises et qui contribuent à la formation des futurs enseignants quand ils réalisent le stage d'enseignement. D'une autre part, nous voulons explorer s'ils se sentent préparés et formés pour mener à bien le rôle de responsable de stage.

Les objectifs spécifiques sont les suivants:

- Déterminer quelles sont les compétences qui doivent être acquises pour former au futur enseignant.

- Contribuer à la formation des enseignants mentors pour l'accomplissement de leurs fonctions.

Dans l'étude on a utilisé une méthodologie à caractère quantitative. On a élaboré un questionnaire avec une échelle de type Likert qui a été appliqué aux enseignants mentors des écoles de la Communauté de Madrid afin de recueillir autant d'informations que possible sur les compétences spécifiques accomplies par des enseignants mentors pendant le stage des futurs enseignants. 105 écoles ont participé pendant l'année 2012-2013. L'échantillonnage participant réel d'enseignants mentors se compose de 674 personnes. La fiabilité du questionnaire a été obtenue par l'alpha de Cronbach avec une fiabilité de 0,859 dans les blocs: l'enseignant mentor et l'école d'une part et, d'autre part, l'enseignant mentor et le développement des compétences spécifiques d'enseignement des stagiaires. Le programme statistique spss 20.0 a été utilisé pour l'analyse des résultats, le test de contraste en utilisant ANOva et pour l'analyse de segmentation a été utilisé la méthode de croissance cHaid pour déterminer le niveau de satisfaction des enseignantes mentors en ce qui concerne leur formation et la compétence pour exercer son rôle d'enseignant mentor.

Parmi les résultats obtenus on peut souligner l'importance de la relation entre l'enseignant mentor et le stagiaire d'une part, en établissant un climat de confiance et d'assistance qui facilitera leur développement professionnel, et d'autre part, il est prouvé le professionnalisme et les compétences qui ont les enseignants mentors dans l'exercice de leur travail. Comme conclusions et propositions futures de formation, il est prouvé le besoin de donner aux enseignants mentors une formation continue afin de faciliter l'accomplissement de leurs tâches pendant l'accompagnement de l'élève stagiaire.

Mots clés: mentor; tuteur; compétences; practicum; stage; formation.

\section{INTRODUCCIÓN}

El objetivo principal de las prácticas de enseñanza es la cualificación profesional de quienes participan en ellas, estudiantes de magisterio futuros maestros, 
maestros titulares de aulas en los centros y profesores de universidad. Por ello, consideramos que para los estudiantes de magisterio la principal finalidad consiste en prepararse, entrenarse e iniciarse en el ejercicio de ser maestro y para los maestros responsables del aula que desempeñan la función de mentor de alumnos en prácticas, la finalidad primordial es doble. Por una parte consiste en cooperar en la formación de los futuros docentes en los aspectos de la práctica educativa y didáctica en el aula y en el centro y, por otra parte, les capacita y cualifica a nivel profesional.

La práctica de la enseñanza se considera fundamental dentro del proceso de formación docente del futuro maestro porque durante las mismas se lleva a término el contraste de la teoría pedagógica con la realidad educativa contribuyendo a tomar conciencia del rol que le corresponderá desempeñar en la institución escolar.

El periodo de prácticas de enseñanza, conocido como Prácticum, requiere del trabajo colaborativo de diferentes agentes formadores. Dentro de la universidad, profesores tutores y en los centros educativos, maestros mentores. Esto nos lleva a plantear el siguiente problema de investigación. ¿Cuál es el rol del mentor? ¿Qué características profesionales posee? ¿Qué competencias debe tener adquiridas para el desarrollo de su quehacer profesional con respecto al alumno en prácticas?

En este artículo se analiza la figura del maestro mentor, sus funciones y competencias profesionales y cómo esto incide en la formación del estudiante de magisterio en prácticas. Dedicando una especial atención a aquellas competencias en las que se siente mejor formado para el desarrollo de su labor de mentor.

Se utiliza una metodología cuantitativa. Mediante un cuestionario se ha recogido la opinión de los maestros mentores de educación infantil y primaria de los centros educativos con el objeto de determinar las competencias específicas de los maestros mentores en ejercicio. Se finaliza con la presentación de los principales resultados que permiten elaborar conclusiones y propuestas futuras de formación.

\section{EL PROFESOR MENTOR DE PRÁCTICAS}

Hablar de mentoría o mentorización no es un concepto nuevo. Por mentoría se entiende una relación de desarrollo personal en la que una persona más experimentada, o con mayor conocimiento, en este caso el tutor de aula o maestro de escuela ayuda a otros menos experimentados o con menor conocimiento, el alumno en prácticas, a lograr sus metas, cultivar sus habilidades y desarrollar sus capacidades profesionales. Se desarrolla en el contexto del trabajo en donde el aprendiz de maestro está aprendiendo un rol laboral (Wisker, Exley, Antoniu y Ridley, 2012: 20).

En este caso utilizamos el término de "mentor" para hacer referencia a aquellos maestros que realizan las funciones de tutores en los centros de prácticas, diferenciándolos de los tutores de universidad. Según la RAE mentor significa «Consejero o guía» (DRAE, 2001: 1489). 
En este sentido se convierte en un instructor dando la oportunidad a los estudiantes de poder interactuar en el aula con los alumnos de infantil o primaria, y al mismo tiempo que éstos puedan aprender viendo y haciendo. Debido a su experiencia enseña, aconseja, guía y ayuda (Moon, 1996: 67) a desarrollarse al futuro maestro.

En esta investigación nos centramos en la figura del mentor, maestro de escuela y más en concreto en las competencias clave que definen su quehacer profesional ya que es la persona experimentada del centro educativo que orientará, guiará y asesorará en la formación del alumno de magisterio en prácticas. «Es la primera imagen del mundo profesional que recibe el estudiante... facilita la integración del estudiante en prácticas en la organización que aprende y le facilita la inserción profesional... observa, verifica, ofrece orientaciones para mejorar las actividades del estudiante» (Martínez y Raposo, 2011: 155 y 159-160).

Consideramos, por tanto, necesario identificar el rol del mentor, sus características profesionales y determinar las competencias que debe tener adquiridas para ejercer su labor.

Existen en esta línea algunos estudios que han abordado estas cuestiones y han tratado de caracterizar los roles que debe asumir a la hora de ejercer sus funciones. Autores como Cid, Pérez y Sarmiento (2011), Martínez Figueira y Raposo (2011) y Zabalza (2011) sistematizan las funciones que realizan dichos agentes: acoger al estudiante, facilitarle el conocimiento del contexto y del aula, comunicarse y coordinarse con otros tutores, supervisar y orientar las intervenciones del alumno en el aula, observar sus actuaciones, realizar el seguimiento y evaluarlo, facilitar la participación del estudiante en la dinámica del aula...

\section{FunCiONES DEL MAESTRO MENTOR DE PRÁCTICAS}

Actualmente las funciones del maestro mentor de prácticas vienen determinadas en la O. 10346/2012 de 11 de septiembre de la Consejería de Educación de la Comunidad de Madrid, en la que se establece un protocolo para la adscripción y realización de las prácticas de los estudiantes de Magisterio (BOEм n. ${ }^{\circ}$ 222: 56-58).

\footnotetext{
Este programa exige, por una parte, designar los centros que durante el mencionado curso escolar participarán en el mismo y, por otra, establecer el procedimiento para que los Profesores y Maestros tutores conozcan el plan de prácticas que van a desarrollar sus tutelados, reciban unas orientaciones específicas para el mejor desempeño de su formación y obtengan el reconocimiento profesional por esta tarea.
}

Esta orden determina una serie de requisitos indicando que «los Maestros o Profesores que ejerzan como Tutores (en este artículo los denominamos Mentores) deberán contar con, al menos, tres años de experiencia docente», así como una serie de funciones que deberán desarrollar durante el periodo en que tengan en sus aulas a alumnos en prácticas:

Serán funciones del tutor (mentor) de prácticas: 
1. Acoger a los alumnos en prácticas en los periodos que se establezcan a lo largo del curso escolar.

2. Posibilitar la iniciación en la práctica docente de los citados alumnos.

3. Asesorar a los alumnos en prácticas en cuestiones pedagógicas y didácticas.

4. Evaluar el desarrollo de las prácticas de los alumnos siguiendo, para ello, los criterios y pautas del plan de prácticas de la Escuela o Facultad Universitaria correspondiente.

Actualmente al mentor le atribuimos otras cualidades, funciones y competencias como: compromiso, coherencia, afinidad a los objetivos de la educación y del centro educativo, profesionalidad, responsabilidad, necesidad e inquietud de mejora. El mentor se implica en las vivencias, en las relaciones personales con sus alumnos, con las familias, con el estudiante en prácticas.

Debe ser consejero, amigable, cooperador. Podríamos ampliar la lista de características: empatía, asertividad, que sea objetivo, "sabio" y discreto. Con un comportamiento moral y ético, coherente entre el pensar y el hacer. Es el maestro que ayuda a otro futuro maestro. Esto trae consigo grandes beneficios en la formación del estudiante en prácticas pues con la ayuda del mentor adquiere mayor confianza en sí mismo, claridad en sus objetivos profesionales futuros, oportunidad para desarrollar sus capacidades y nuevas aptitudes y mayor capacidad para comunicarse en el ámbito profesional.

No cabe duda de que disponer de una buena formación, variadas experiencias profesionales, años de docencia y buenas actitudes hacia la mentorización del estudiante en prácticas será una pieza clave para ser un buen mentor de prácticas.

\section{CONCEPTO DE COMPETENCIA}

Existe diversidad de opiniones respecto al término de competencias. Algunos las sitúan como una serie de habilidades o destrezas, hasta capacidades o aptitudes que una persona pone en funcionamiento para resolver situaciones más o menos complejas y específicas.

Este término, surgido inicialmente en un contexto vinculado a la formación y el empleo, viene utilizándose cada vez más en el campo educativo. Vamos a referirnos a continuación a algunos estudios e informes sobre el tema.

El proyecto de la ocDe denominado DeSeCo (2001) Definición y Selección de Competencias. Define la competencia, como «la capacidad de responder a demandas complejas y llevar a cabo tareas diversas de forma adecuada. Supone una combinación de habilidades prácticas, conocimientos, motivación, valores éticos, actitudes, emociones y otros componentes sociales y de comportamiento que se movilizan conjuntamente para lograr una acción eficaz».

Le Bobterf (2000: 87) considera que competencia es la secuencia de acciones que combinan varios conocimientos, un esquema operativo transferible a una familia de situaciones. 
En palabras de Cano (2005: 14-20), un profesional es competente cuando «sabe manejar y gestionar estas acciones, sabe gestionar con pertinencia, sabe combinar los recursos y movilizarlos en un contexto, sabe transferir, sabe aprender, sabe comprometerse».

De aquí que las competencias no las podamos reducir a un simple saber o saber hacer. Implican a las capacidades, sin las que sería muy difícil llegar a ser competente. Es un saber que se aplica, susceptible de adecuarse a una diversidad de contextos y tiene un carácter integrador, abarcando conocimientos, procedimientos y actitudes.

En términos de Perrenoud (2007), competencia es «la capacidad de movilizar varios recursos cognitivos para hacer frente a un tipo de situaciones", por tanto, las competencias no son en sí mismas conocimientos, habilidades, actitudes y prácticas, si bien el desarrollo de estos recursos es lo que se precisa para ser un profesional competente.

En este sentido las competencias profesionales se crean y se adquieren mediante el ejercicio y experiencia profesional. De ahí que un profesional es competente cuando es reconocidamente bueno en su práctica profesional (OIт: 2008 y Perrenoud: 2007).

Para Zabala (2008: 31), competencia es «la alternativa a modelos formativos que sirven para dar respuesta a los problemas cotidianos». Desde esta perspectiva podemos observar que las respuestas van ligadas a la eficiencia del ser humano para resolver problemas. Las competencias no son una panacea (Coll, 2007), no provienen de un modelo teórico, más bien es el uso y aplicación específica que en función de las necesidades queremos darle. Así el concepto competencia será «la capacidad o habilidad de efectuar tareas o hacer frente a situaciones diversas de forma eficaz en un contexto determinado y para ello es necesario movilizar actitudes, habilidades y conocimiento al mismo tiempo y de forma intencionada" (Zabala y Arnau, 2008: 43-44), citado por Guzmán Ibarra y Marín Uribe (2011).

\section{COMPETENCIAS DEL MENTOR}

Teniendo en cuenta que las competencias profesionales son aquellas adquiridas en el ejercicio de la experiencia profesional, consideramos que un profesional es competente cuando es reconocidamente bueno en su práctica profesional. En palabras de Zabalza (2001) sirven para definir el sentido y los contenidos de la formación de una forma práctica y referida al ejercicio profesional, por lo que no pueden entenderse al margen del contexto en el que se desarrollan.

En el Título de Grado de Magisterio (aneca, 2005) se identifican cuatro competencias específicas de formación disciplinar y profesional común a todos los perfiles de maestros: competencia cognitiva (saber), competencia funcional (saber hacer), competencia personal (saber estar) y competencia ética (saber ser). Un mentor competente será aquel que conoce y sistematiza sus procesos de construcción 
del conocimiento; sabe cómo intervenir, pues posee habilidades y destrezas cognitivas; sabe cómo relacionarse, cómo utilizar estratégicamente el conocimiento.

Las competencias del mentor en cierto modo están condicionadas por las competencias que deben adquirir los estudiantes de magisterio y que vienen recogidas en la Orden ECI/3854/2007 y ECI/3857/2007, de 27 de diciembre, por la que se establecen los requisitos para la verificación de los títulos universitarios que habiliten para el ejercicio de la profesión de Maestro en Educación Infantil y Educación Primaria, respectivamente tal y como quedan recogidas en la Tabla 1. Van encaminadas a formar un profesional que sea capaz de gestionar y manejar situaciones complejas, que sepa actuar y reaccionar adecuadamente utilizando los recursos necesarios en un contexto determinado, que sepa comprender, reflexionar y transferir conocimientos.

TABLA 1

Competencias que deben adquirir los estudiantes de Magisterio

\begin{tabular}{|c|c|}
\hline \multicolumn{2}{|c|}{ COMPETENCIAS GRADO DE MAGISTERIO } \\
\hline EDUCACIÓN INFANTIL & EDUCACIÓN PRIMARIA \\
\hline $\begin{array}{l}\text { 1. Conocer los objetivos, contenidos } \\
\text { curriculares y criterios de evaluación de la } \\
\text { educación infantil. }\end{array}$ & $\begin{array}{l}\text { 1. Conocer las áreas curriculares de } \\
\text { la educación primaria, la relación } \\
\text { interdisciplinar entre ellas, los criterios de } \\
\text { evaluación y el cuerpo de conocimientos } \\
\text { didácticos. }\end{array}$ \\
\hline $\begin{array}{l}\text { 2. Promover y facilitar los aprendizajes en } \\
\text { la primera infancia, desde una perspectiva } \\
\text { globalizadora e integradora. }\end{array}$ & $\begin{array}{l}\text { 2. Diseñar, planificar y evaluar procesos } \\
\text { de enseñanza y aprendizaje, tanto } \\
\text { individualmente como en colaboración con } \\
\text { otros docentes y profesionales del centro. }\end{array}$ \\
\hline $\begin{array}{l}\text { 3. Diseñar y regular espacios de aprendizaje } \\
\text { en contextos de diversidad que atiendan } \\
\text { a las singulares necesidades educativas de } \\
\text { los estudiantes, a la igualdad de género, } \\
\text { a la equidad y al respeto de los derechos } \\
\text { humanos. }\end{array}$ & $\begin{array}{l}\text { 3. Abordar con eficacia situaciones de } \\
\text { aprendizaje de lenguas en contextos } \\
\text { multiculturales y plurilingües. Fomentar la } \\
\text { lectura y el comentario crítico de textos de } \\
\text { los diversos dominios científicos y culturales. }\end{array}$ \\
\hline $\begin{array}{l}\text { 4. Fomentar la convivencia en el aula y fuera } \\
\text { de ella y abordar la resolución pacífica de } \\
\text { conflictos. Saber observar sistemáticamente } \\
\text { contextos de aprendizaje y convivencia y } \\
\text { saber reflexionar sobre ellos. }\end{array}$ & $\begin{array}{l}\text { 4. Diseñar y regular espacios de aprendizaje } \\
\text { en contextos de diversidad y que atiendan } \\
\text { a la igualdad de género, a la equidad y } \\
\text { al respeto de los derechos humanos que } \\
\text { conformen los valores de la formación } \\
\text { ciudadana. }\end{array}$ \\
\hline
\end{tabular}




\begin{tabular}{|c|c|}
\hline \multicolumn{2}{|c|}{ COMPETENCIAS GRADO DE MAGISTERIO } \\
\hline EDUCACIÓN INFANTIL & EDUCACIÓN PRIMARIA \\
\hline $\begin{array}{l}\text { 5. Reflexionar en grupo sobre la } \\
\text { aceptación de normas y el respeto a } \\
\text { los demás. Promover la autonomía } \\
\text { y la singularidad de cada estudiante } \\
\text { como factores de educación de las } \\
\text { emociones, los sentimientos y los } \\
\text { valores en la primera infancia. }\end{array}$ & $\begin{array}{l}\text { 5. Fomentar la convivencia en el aula } \\
\text { y fuera de ella, resolver problemas de } \\
\text { disciplina y contribuir a la resolución } \\
\text { pacífica de conflictos. Estimular y } \\
\text { valorar el esfuerzo, la constancia y la } \\
\text { disciplina personal en los estudiantes. }\end{array}$ \\
\hline $\begin{array}{l}\text { 6. Conocer la evolución del lenguaje en la } \\
\text { primera infancia, saber identificar posibles } \\
\text { disfunciones y velar por su correcta } \\
\text { evolución. Abordar con eficacia situaciones } \\
\text { de aprendizaje de lenguas en contextos } \\
\text { multiculturales y multilingües. Expresarse } \\
\text { oralmente y por escrito y dominar el uso } \\
\text { de diferentes técnicas de expresión. }\end{array}$ & $\begin{array}{l}\text { 6. Conocer la organización de los colegios } \\
\text { de educación primaria y la diversidad } \\
\text { de acciones que su funcionamiento } \\
\text { comprende. Desempeñar las funciones de } \\
\text { tutoría y de orientación con los estudiantes } \\
\text { y sus familias, para atender las singulares } \\
\text { necesidades educativas de los estudiantes. } \\
\text { Asumir que el ejercicio de la función } \\
\text { docente ha de ir perfeccionándose y } \\
\text { adaptándose a los cambios científicos, } \\
\text { pedagógicos y sociales a lo largo de la vida. }\end{array}$ \\
\hline $\begin{array}{l}\text { 7. Conocer las implicaciones educativas } \\
\text { de las tecnologías de la información } \\
\text { y la comunicación y, en particular, de } \\
\text { la televisión en la primera infancia. }\end{array}$ & $\begin{array}{l}\text { 7. Colaborar con los distintos sectores de } \\
\text { la comunidad educativa y del entorno } \\
\text { social. Asumir la dimensión educadora de } \\
\text { la función docente y fomentar la educación } \\
\text { democrática para una ciudadanía activa. }\end{array}$ \\
\hline $\begin{array}{l}\text { 8. Conocer fundamentos de dietética e } \\
\text { higiene infantiles. Conocer fundamentos } \\
\text { de atención temprana y las bases y } \\
\text { desarrollos que permiten comprender } \\
\text { los procesos psicológicos, de aprendizaje } \\
\text { y de construcción de la personalidad. }\end{array}$ & $\begin{array}{l}\text { 8. Mantener una relación crítica y autónoma } \\
\text { respecto de los saberes, los valores y las } \\
\text { instituciones sociales públicas y privadas. }\end{array}$ \\
\hline $\begin{array}{l}\text { 9. Conocer la organización de las } \\
\text { escuelas de educación infantil y } \\
\text { la diversidad de acciones que su } \\
\text { funcionamiento comprende. }\end{array}$ & $\begin{array}{l}\text { 9. Valorar la responsabilidad individual } \\
\text { y colectiva en la consecución } \\
\text { de un futuro sostenible. }\end{array}$ \\
\hline $\begin{array}{l}\text { 10. Actuar como orientador de padres } \\
\text { y madres en relación con la educación } \\
\text { familiar en el periodo de } 0-6 \text { años } \\
\text { y dominar habilidades sociales. }\end{array}$ & $\begin{array}{l}\text { 10. Reflexionar sobre las prácticas de } \\
\text { aula para innovar y mejorar la labor } \\
\text { docente. Adquirir hábitos y destrezas para } \\
\text { el aprendizaje autónomo y cooperativo } \\
\text { y promoverlo entre los estudiantes. }\end{array}$ \\
\hline
\end{tabular}




\begin{tabular}{|l|l|}
\hline \multicolumn{2}{|c|}{ COMPETENCIAS GRADO DE MAGISTERIO } \\
\hline \multicolumn{1}{|c|}{ EDUCACIÓN INFANTIL } & \multicolumn{1}{c|}{ EDUCACIÓN PRIMARIA } \\
\hline $\begin{array}{l}\text { 11. Reflexionar sobre las prácticas de } \\
\text { aula para innovar y mejorar la labor } \\
\text { docente. Adquirir hábitos y destrezas para } \\
\text { el aprendizaje autónomo y cooperativo } \\
\text { y promoverlo en los estudiantes. }\end{array}$ & $\begin{array}{l}\text { 11. Conocer y aplicar en las aulas las } \\
\text { tecnologías de la información y de la } \\
\text { comunicación. Discernir selectivamente la } \\
\text { información audiovisual que contribuya } \\
\text { a los aprendizajes, a la formación } \\
\text { cívica y a la riqueza cultural. }\end{array}$ \\
\hline $\begin{array}{l}\text { 12. Comprender la función, las } \\
\text { posibilidades y los límites de la educación } \\
\text { en la sociedad actual y las competencias } \\
\text { fundamentales que afectan a los colegios } \\
\text { de educación infantil y a sus profesionales. } \\
\text { Conocer modelos de mejora de la calidad } \\
\text { con aplicación a los centros educativos. }\end{array}$ & $\begin{array}{l}\text { 12. Comprender la función, las } \\
\text { posibilidades y los límites de la educación } \\
\text { en la sociedad actual y las competencias } \\
\text { fundamentales que afectan a los colegios de } \\
\text { educación primaria y a sus profesionales. } \\
\text { Conocer modelos de mejora de la calidad } \\
\text { con aplicación a los centros educativos. }\end{array}$ \\
\hline
\end{tabular}

Fuente: Elaboración propia.

En la Orden 10346/2012, de 11 de septiembre, citada anteriormente, se establece el procedimiento que se ha de seguir para que los alumnos de Magisterio y especialidades relacionadas con la enseñanza, matriculados en Escuelas Universitarias de Formación del Profesorado y Facultades durante el curso 2012-2013, puedan realizar las prácticas en centros de la Comunidad de Madrid (BOCM 222: 56):

permitirá a los alumnos de las Escuelas Universitarias de Formación del Profesorado y Facultades su iniciación en la práctica docente directa y en la orientación psicopedagógica, además de conocer los aspectos pedagógicos, organizativos y de funcionamiento de los centros con el apoyo y bajo la tutela de Profesores y Maestros en ejercicio que reúnen las características y preparación adecuadas.

Cabe destacar que, tal y como se refleja en la orden, se establece que para poder ser mentor los maestros deberán contar con, al menos, tres años de experiencia y siguiendo esta línea la Consejería de Educación, a través de la Dirección General de Mejora de la Calidad de la Enseñanza, convocó durante el mes de febrero de 2013 un curso de Formación para Tutores del Prácticum. Los objetivos perseguidos en ese curso fueron que los asistentes adquiriesen las competencias adecuadas para el desempeño de las tareas que requiere en el proceso de tutela de los estudiantes de Magisterio. Contó con la participación de ponentes de distintas universidades de Madrid. Los contenidos que se abordaron a lo largo de cuatro sesiones fueron:

- La figura del tutor (mentor) del Prácticum.

- Competencias a desarrollar durante el proceso del Prácticum: competencias profesionales y competencias comunicativas. 
- Aspectos organizativos y de funcionamiento del centro y organización del Prácticum de cada universidad.

- Evaluación del Prácticum: estudiantes, plan y universidad.

La evaluación de los participantes en el curso fue altamente positiva. Como mejoras para el desarrollo del Prácticum destacan que se debería aumentar la comunicación entre el profesor tutor de la universidad con el maestro mentor del centro educativo, unificar las evaluaciones de los estudiantes en todas las universidades y mantener más contacto e información entre las universidades.

En otros estudios realizados por Sánchez-Núñez, Ramírez Fernández y García Guzman (2011: 125) de la Universidad de Granada sobre la formación de estos profesionales proponen los siguientes objetivos formativos: "Crear un foro de formación, intercambio y debate sobre la enseñanza, reflexionar y consensuar un modelo de buenas prácticas externas, contribuir a la formación de tutores externos de Prácticas, realizar acciones de colaboración y coordinación entre universidades y los centros educativos y elaborar planes de acogida al estudiante en prácticas».

Del estudio llevado a cabo por Ríos Ariza y Gómez Barajas (2013: 183) sobre la relación entre competencias básicas de los estudiantes y las competencias del profesorado, se desprende que aquellos docentes críticos, reflexivos, en definitiva competentes, serán una referencia clave en la orientación de las competencias que deban adquirir los estudiantes para su formación como docentes. En concreto señala el "tratamiento de la información y competencia digital» y la "participación social y ciudadana", lo que propiciará entornos de aprendizaje encaminados a la formación de ciudadanos críticos, libres, capaces de utilizar las Tic en todos los ámbitos de la vida.

Por tanto, es tarea del tutor-mentor facilitar la adquisición de competencias y en "la práctica tutorial, habitual en los centros, pueden realizar una contribución importante al logro de las competencias asociadas al autocontrol de los aprendizajes, al desarrollo afectivo y emocional o a la adquisición de habilidades sociales, a la competencia para aprender a aprender y a la autonomía e iniciativa personal» (Tiana, 2011: 72), competencias básicas del currículo de educación primaria que los alumnos de esta etapa deben alcanzar y que los estudiantes de Magisterio deben contribuir a su desarrollo.

En el estudio llevado a cabo por Inostroza de Celis, Jara y Tagle (2010: 122123), se realiza un análisis sobre el perfil del mentor basado en competencias que consideramos valioso para nuestro trabajo. Destacando entre otras las siguientes:

- Acompaña en la construcción de los saberes asociados a la cultura escolar (mediación profesional y gestión).

- Organiza y desarrolla procesos de andamiaje, enfocados en situaciones de la vida real y de la experiencia del profesor principiante (en nuestro caso, estudiante de Magisterio en prácticas). 
- Permite al profesor principiante (estudiante de magisterio) afianzar habilidades de su propio desempeño profesional asumiendo aciertos, errores y debilidades.

- Se caracteriza por ser un profesional que moviliza recursos propios y ajenos a sus contextos de desempeño.

- Diseña, organiza, implementa acciones docentes.

En definitiva, el profesor-mentor debe facilitar el desarrollo de las competencias profesionales en el estudiante de magisterio; requerirá construir conocimientos y desarrollar habilidades, destrezas y actitudes que van más allá de aquellas necesarias para ejercer el rol profesional.

\section{Metodología}

La metodología que se utiliza en este estudio es de carácter cuantitativo. Para la recogida de datos se empleó como instrumento el cuestionario elaborado con escala tipo Likert.

Para identificar las competencias profesionales que deben tener asumidas y adquiridas los mentores y que contribuyen a la formación de los futuros docentes noveles, estudiantes en prácticas de Magisterio, y explorar cuáles son sus necesidades formativas para ejercer esta función, se aplicó un cuestionario diseñado para esta ocasión. Se estructuró en torno a cuatro bloques: el primero recoge aspectos relativos a las características personales; el segundo a los contenidos del Prácticum en Magisterio; el tercero, el mentor y su relación con el tutor universitario, y el cuarto presenta las competencias básicas del mentor en relación a las competencias que deben contribuir a desarrollar en el estudiante en prácticas. En este estudio nos hemos centrado en el primer y cuarto bloque.

Los objetivos e interrogantes específicos se centran en:

- Determinar cuáles son las competencias que deben tener asumidas y adquiridas para formar al futuro maestro.

- Contribuir a la formación de los maestros mentores para el desempeño de sus funciones.

El proceso de esta investigación se llevó a cabo durante los meses de febrero y marzo de 2013. Los cuestionarios se remitieron fotocopiados a los maestros mentores de 105 colegios de la Comunidad de Madrid, de los cuales 23 eran colegios públicos, 75 concertados y 7 privados. Se enviaron 733 cuestionarios a los que respondieron 674 mentores. Respecto a la validez del contenido se ha recurrido a la opinión de expertos vinculados al campo de las prácticas de enseñanza.

En lo que se refiere a la fiabilidad del instrumento se llevó a cabo mediante el paquete estadístico spss 20.0. Concretamente se ha utilizado el subprograma RELIABILITY ANALYSIS-SCALE (ALPHA), obteniendo mediante el coeficiente alpha de Cronbach un valor de 0,859 , lo que indica un nivel elevado de fiabilidad. Para 
el análisis inferencial, la prueba de contraste mediante anova y para el análisis de segmentación se utilizó el método de crecimiento cHAID para identificar el nivel de satisfacción de los mentores con respecto a su formación y capacidad para ejercer su función de mentor.

\section{DESCRIPCIÓN DE RESUltados}

Se trataba de recoger datos de toda la población y si bien se produjeron algunas pérdidas, la participación de los sujetos implicados fue muy alta situándose en un $92 \%$. Del total de la muestra, el $78 \%$ de los mentores son mujeres y el $22 \%$ hombres. La edad de la muestra es relativamente joven ya que el 42,3\% se sitúan entre los 30-39 años, el 23,4\% entre los 40-49 años y el resto son menores de 30 y mayores de 50 años. El 42,9\% de los encuestados llevan desempeñando la tarea de mentor poco tiempo, entre 5 o menos años, seguidos de aquellos que llevan realizando esta tarea entre 6 y 10 años, lo que supone un 21,7\%. Se les preguntó si se "sentían preparados y cualificados para ejercer su labor de mentor de Prácticas", a lo que respondieron que un $97,4 \%$ se sienten bastante y muy cualificados para ejercer su tarea de mentor.

En el bloque IV del cuestionario se recogen preguntas que hacen referencia a la tutela del maestro mentor con el fin de determinar si facilita el desarrollo de competencias específicas comunes que deben adquirir los estudiantes en prácticas durante su estancia en el centro educativo. Debemos tener en cuenta que las competencias del mentor, como orientador y guía, influyen o inciden, de alguna forma, en la adquisición de competencias profesionales de los estudiantes de Magisterio. Los resultados han puesto de relieve que la labor ejercida por el mentor se considera como un elemento importante en la formación del alumno en prácticas con un $98,2 \%$ una vez agrupados los valores de bastante y mucho. En relación con la cuestión relativa a si se consideran como un «modelo docente», los resultados muestran que son plenamente conscientes de su rol en un 92,4\%. Sin embargo, alcanza un menor porcentaje el ítem relativo al reconocimiento profesional de su «labor de maestro mentor", obteniendo un valor agrupado (nada y poco) de un 79,3\%.

En cuanto a la pregunta de si «el mentor ayuda a desarrollar en el alumno la capacidad de comprender los procesos educativos en general y los de enseñanzaaprendizaje en particular», respondieron mayoritariamente en un 99,1\%, que bastante y mucho, con lo que ponemos de manifiesto que los mentores tienen en alta consideración su labor en el desarrollo del Prácticum.

En esta línea, ante la pregunta «el mentor se preocupa de que el alumno incremente el dominio de las capacidades básicas y profesionales de la función docente», el 99,1\% considera que bastante y mucho. Analizando las medias obtenidas en este apartado, la puntuación media otorgada muestra una valoración muy alta, por encima del 3,49 en un recorrido de 1 a 4 puntos.

Por otra parte, consideramos que la labor del mentor debería posibilitar al estudiante en prácticas la adquisición de competencias profesesionales que le 
permitan alcanzar una formación adecuada para ejercer como futuro maestro. Ser capaces de alcanzar una buena formación científico-cultural y tecnológica, analizar y diseñar proyectos educativos, construir materiales didácticos y procurar un uso adecuado y autónomo en los marcos específicos de las distintas disciplinas y currículos de las etapas de educación infantil y educación primaria. En el informe de las medias obtenido pudimos apreciar que preparar, seleccionar o construir materiales didácticos y utilizarlos en los marcos específicos de las distintas disciplinas e iniciar un desarrollo profesional autónomo obtuvieron las medias más altas, entre 3,11 y 3,24 .

Los mentores respondieron en un 56,7\% que posibilitan bastante y mucho en los estudiantes de magisterio la capacidad de alcanzar una formación científico-cultural y tecnológica; el 65,8\% consideraron que adquirirían la capacidad de analizar y cuestionar las propuestas curriculares; con respecto a si obtendrían la capacidad de diseñar y elaborar proyectos educativos y unidades de programación, el $72 \%$ respondió que bastante y mucho; el 85,2\% opinó que sí adquieren la capacidad de integrar los saberes disciplinares, transversales y multidisciplinares adecuados al respectivo nivel educativo en el que realicen sus prácticas; respondieron en un 90,9\% que los estudiantes alcanzarían la capacidad de preparar, seleccionar o construir materiales didácticos, y en un 89,4\% que adquirirían la capacidad de iniciar un desarrollo profesional autónomo.

Teniendo en cuenta estas dos últimas respuestas queda constatado que los mentores consideran muy positivamente que los estudiantes en prácticas «adquieran ciertas capacidades profesionales», gracias al ejercicio de su labor como mentor.

Por otra parte, el estudiante en prácticas también tiene que desarrollar ciertas capacidades, que ya posee, pero que se espera aumentar con la ayuda y colaboración del mentor. En esta ocasión se les preguntó por el desarrollo de las siguientes competencias: enseñar contenidos de forma comprensible, potenciar el rendimiento de los alumnos y su progreso escolar en el marco de una educación integral, respetar las diferencias culturales y personales de los alumnos y prever necesidades y demandas educativas, asumir responsabilidades y tomar decisiones desarrollando y asumiendo la dimensión ética de ser maestro y ser capaz de relacionarse y mantener el equilibrio emocional en diversas situaciones.

En el informe de puntuaciones medias con respecto a estas competencias se constató que existen pocas diferencias entre ellas, siendo todas muy altas (entre 3,23 y 3,49) en un recorrido de 1 a 4 puntos.

En un segundo momento se utilizó la prueba de ANOva para constatar si existían diferencias significativas entre estas competencias que permiten el desarrollo de la labor del mentor y los años que llevaban ejerciendo el magisterio los mentores, viendo que existen diferencias significativas en las competencias relacionadas con la responsabilidad y comunicación en el ejercicio docente. Así el valor del estadístico de contraste «F» es más elevado en "asumir responsabilidades y tomar decisiones» (F- 4,426) y "relacionarse, comunicarse y mantener el equilibrio emocional en diversas situaciones» (F- 3,112), es menos valorada por los mentores que 
llevan ejerciendo el magisterio entre 6 y 10 años obteniendo una media de 3,24, mientras que en el colectivo entre 11 y 15 años, con una media de 3,49 es el que ofrece una opinión más positiva. Ver Tabla 2 y Gráficos 1 y 2.

TABLA 2

Competencias del mentor

\begin{tabular}{|l|c|c|c|c|c|c|c|c|}
\hline \multicolumn{1}{|c|}{ CompetencIas } & GLOBAL & $\begin{array}{c}50 \\
\text { MENOS }\end{array}$ & $\begin{array}{c}\text { ENTRE } \\
6 \text { Y } 10\end{array}$ & $\begin{array}{c}\text { ENTRE } \\
11 \text { Y } 15\end{array}$ & $\begin{array}{c}\text { ENTRE } \\
16 \text { Y } 20\end{array}$ & $\begin{array}{c}\text { MÁs } \\
\text { DE } 20\end{array}$ & «F” & $\begin{array}{c}\text { SIGNFICA- } \\
\text { TIVIDAD }\end{array}$ \\
\hline $\begin{array}{l}\text { Enseñar contenidos de } \\
\text { forma comprensible }\end{array}$ & 3,41 & 3,52 & 3,33 & 3,50 & 3,41 & 3,38 & 2,397 &, 049 \\
\hline $\begin{array}{l}\text { Respetar las diferencias } \\
\text { culturales personales } \\
\text { de los alumnos y } \\
\text { demás miembros de la } \\
\text { comunidad educativa }\end{array}$ & 3,49 & 3,47 & 3,50 & 3,55 & 3,51 & 3,43 & 1,025 &, 394 \\
\hline $\begin{array}{l}\text { Utilizar e incorporar } \\
\text { adecuadamente en } \\
\text { las actividades de } \\
\text { enseñanza-aprendizaje } \\
\text { las tecnologías de } \\
\text { la información y la } \\
\text { comunicación }\end{array}$ & 3,24 & 3,31 & 3,22 & 3,27 & 3,15 & 3,25 &, 598 &, 664 \\
\hline $\begin{array}{l}\text { Relacionarse, } \\
\text { comunicarse y mantener } \\
\text { el equilibrio emocional } \\
\text { en diversas situaciones }\end{array}$ & 3,38 & 3,48 & 3,29 & 3,49 & 3,43 & 3,34 & 3,112 &, 015 \\
\hline $\begin{array}{l}\text { Prever nuevas } \\
\text { necesidades y } \\
\text { demandas educativas }\end{array}$ & 3,23 & 3,26 & 3,20 & 3,36 & 3,21 & 3,16 & 2,141 &, 074 \\
\hline $\begin{array}{l}\text { Asumir responsabilidades } \\
\text { y tomar decisiones }\end{array}$ & 3,31 & 3,43 & 3,24 & 3,49 & 3,33 & 3,22 & 4,426 &, 002 \\
\hline $\begin{array}{l}\text { Asumir la dimensión } \\
\text { ética de ser maestro }\end{array}$ & 3,46 & 3,44 & 3,41 & 3,49 & 3,49 & 3,46 &, 496 &, 739 \\
\hline $\begin{array}{l}\text { Potenciar el rendimiento } \\
\text { de los alumnos y } \\
\text { su progreso escolar, } \\
\text { en el marco de una } \\
\text { educación integral }\end{array}$ & 3,38 & 3,34 & 3,27 & 3,47 & 3,47 & 3,39 & 2,895 &, 022 \\
\hline $\begin{array}{l}\text { Asumir la necesidad de } \\
\text { desarrollo profesional } \\
\text { continuo, mediante } \\
\text { la autoevaluación de } \\
\text { la propia práctica }\end{array}$ & 3,36 & 3,44 & 3,32 & 3,41 & 3,31 & 3,36 &, 880 &, 475 \\
\hline
\end{tabular}


GRÁFICO 1

Relacionarse, comunicarse y mantener el equilibrio emocional

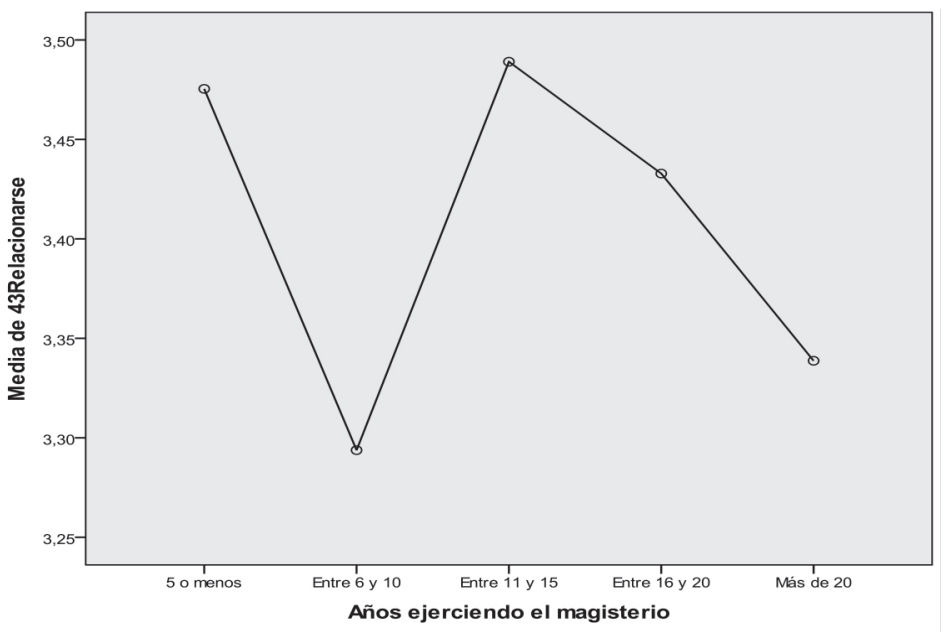

GRÁFICO 2

Asumir responsabilidades y tomar decisiones

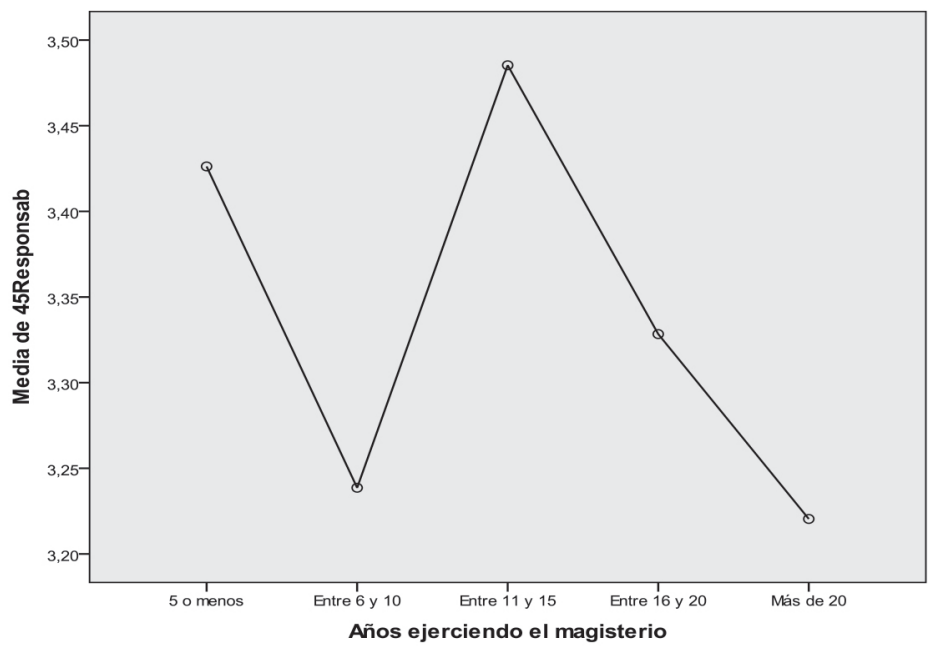

Estos datos nos permiten confirmar que los años de dedicación al magisterio es una variable importante de categorización que influye en la percepción que tienen los mentores sobre su labor profesional con los estudiantes en prácticas. 
Para terminar con el estudio nos interesaba conocer en qué aspectos los mentores se sienten altamente capacitados y formados para ejercer su función de mentor y se les pidió que indicasen en una escala de 1 a 10 (1 mínimo-10 máximo), "cómo se sienten de formados para ejercer su labor como maestro mentor". Se recurrió a un análisis de segmentación. El resultado obtenido muestra que en lo que mejor se sienten formados es en ayudar al alumno en prácticas a diseñar y elaborar proyectos educativos y unidades de programación que les permitan adaptar currículos al contexto sociocultural, potenciando el rendimiento de los alumnos y su progreso escolar en el marco de una educación integral (Gráfico 3).

\section{GRÁFICO 3}

Aspecto en el que mejor se sienten formados los mentores para ejercer su función

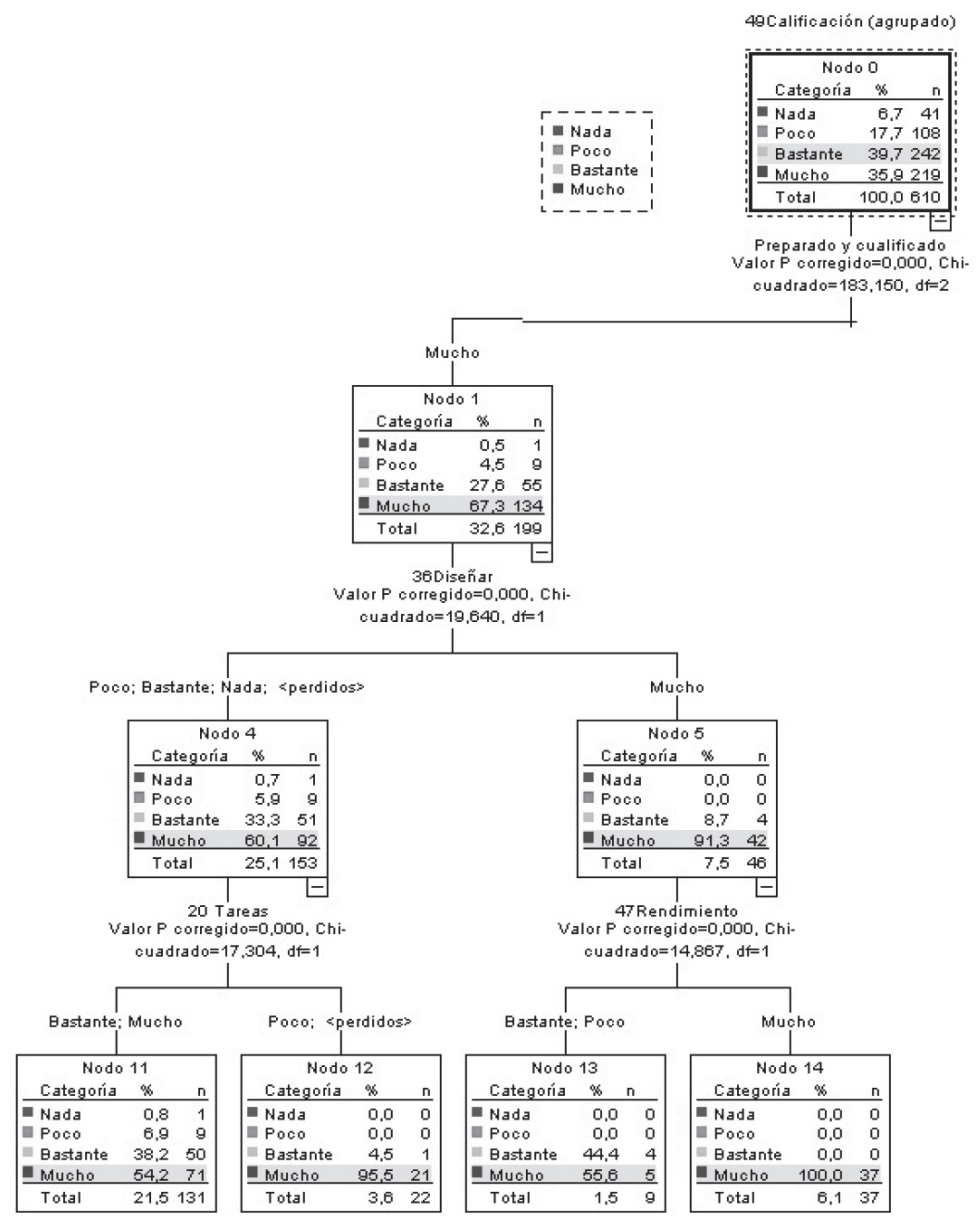




\section{CONCLUSIONES Y PROPUESTAS}

La cualificación profesional del maestro mentor incide directamente en la formación del estudiante en prácticas, por lo que el desarrollo de competencias adecuadas en su acción tutorial se considera imprescindible.

Los resultados hallados en este estudio muestran que los mentores, cuyo perfil es el de mujer entre 30-39 años que trabaja en un colegio concertado en la etapa de educación primaria y lleva ejerciendo como mentor de prácticas en torno a cinco años, son un elemento fundamental en la formación del estudiante en prácticas y éstos los consideran un modelo docente a seguir.

Tienen en gran estima su labor para facilitar el desarrollo de competencias específicas y profesionales del estudiante en prácticas. En lo que se sienten más capacitados es en preparar, seleccionar, construir materiales didácticos y adaptarlos a los contextos y diferentes niveles educativos potenciando el rendimiento de los alumnos y su progreso escolar en el marco de una educación integral. Hecho que constata la profesionalidad de los mentores en el ejercicio de su labor y esto revierte positivamente en el estudiante en prácticas ya que les facilita el desarrollo e integración de competencias tales como planificar, adaptarse a los contextos, autonomía, detectar dificultades en los alumnos, desarrollar competencias socioemocionales, comunicar y motivar.

Nos encontramos ante un nuevo rol docente del mentor ya que afronta su tarea cotidiana de maestro de infantil o primaria, con un alumnado universitario diferente lo que supone que debe ser capaz de acompañarle en este periodo de aprendizaje que es el Prácticum y facilitarle, al mismo tiempo, el desarrollo de competencias académicas y profesionales.

Esto solo se puede conseguir si el mentor asume como parte de su función docente, ser guía, acompañante, facilitador del proceso de aprendizaje del estudiante en prácticas y que éste desarrolle competencias suficientes para aprender a aprender y enseñar.

En este sentido el mentor deja de tener un rol tradicional de gestor o mero transmisor de conocimientos a ser un profesional que genera y crea ambientes de aprendizaje en el que implicará al estudiante en prácticas a desarrollar paulatinamente su autonomía personal en la toma de decisiones, aprendizaje de estrategias y desarrollo de actividades adecuadas.

Sin embargo, el estudio llevado a cabo tiene limitaciones. Por un lado, se les pregunta a los mentores cómo se sienten de formados para ejercer su tarea con los estudiantes de Magisterio, pero por otro lado sería conveniente en investigaciones futuras preguntarles sobre quién o quiénes deberían asumir esta tarea de formación del mentor de prácticas.

Nuestra sociedad, en cambio constante, debe contar con un profesorado abierto a los cambios e innovador, con un conocimiento general actualizado y capaz de hacer cambios profundos en los contenidos adquiridos desde la reflexión crítica y la capacidad de análisis. 
Las instituciones educativas (Universidades, Escuelas de Magisterio, Centros de Formación del Profesorado, etc.) deben jugar un papel importante en la formación de los mentores y facilitar una oferta amplia y al mismo tiempo indispensable para poder guiar, orientar y procurar el desarrollo de competencias profesionales en el estudiante en prácticas de magisterio.

Defendemos que la formación del profesorado debe insertarse en un enfoque humanista de las competencias, que capacite en vincular la teoría y la práctica, que posibilite resolver problemas derivados de la práctica educativa. Si bien hoy en día la formación debe derivar hacia el logro de competencias intelectuales, profesionales, sociales e interpersonales que garanticen el equilibrio emocional y el compromiso con la profesión docente.

\section{REFERENCIAS BIBLIOGRÁFICAS}

Agencia Nacional de Evaluación de la Calidad y Acreditación (2005). Libro Blanco. Título de Grado de Magisterio. Madrid: ANECA.

Cano, E. (2005). Cómo mejorar las competencias de los docentes. Guía para la autoevaluación y el desarrollo de las competencias del profesorado. Barcelona: Graó.

Cid Sabucedo, A.; Pérez Abellas, A. y Sarmiento, J. A. (2011). La tutoría en el prácticum. Revisión de la literatura. Revista de Educación, 354, 127-154.

Coll, C. (2007). Las competencias en la educación escolar: algo más que una moda y mucho menos que un remedio. Aula de Innovación Educativa, 61, 34-39.

Guzmán Ibarra, I. y Marín Uribe, R. (2011). La competencia y las competencias docentes: reflexiones sobre el concepto y la evaluación. Revista Electrónica Interuniveritaria de Formación del Profesorado, 14 (I), 151-163. Descargado el día 16 de diciembre de 2013. http://www.redaluc.org/pdf/2170/217017192012.pdf.

Inostroza de Celis, G.; Jara, E. y Tagle, T. (2010). Perfil del mentor basado en competencias. Revista de Estudios Pedagógicos, XXXVI, 1, 117-129.

http://dx.doi.org/10.4067/s0718-07052010000100006

Le Boterf, G. (2001). Ingeniería de las competencias. Barcelona: Ediciones Gestión 2000.

Martínez Figueira, M. E. y Raposo Rivas, M. (2011). Funciones generales de la tutoría en el prácticum: entre la realidad y el deseo en el desempeño de la acción tutorial. Revista de Educación, 354, 155-181.

Ministerio de Educación y Ciencia (2007). Orden ECI/3854/2007 y ECI/385772007, de 27 de diciembre, por la que se establecen los requisitos para la verificación de los títulos universitarios oficiales que habiliten para el ejercicio de la profesión de Maestro en Educación Infantil y Educación Primaria. BOE, n. ${ }^{\circ} 312$ de 29 de diciembre de 2007.

Moon, B. (1996). La formación de tutores. Concepto y recursos. En M. A. Zabalza Beraza (Coord.). Actas IV Symposium de Prácticas, tomo I (pp. 65-77). Pontevedra: Diputación Provincial de Pontevedra.

OECD (2002). Definition and selection of Competencies (DeSeCo). Resumen ejecutivo. Descargado el día 15 de mayo de 2013. http://www.OECD.org/edu/statistics/deseco.

OIT. Organización Internacional del Trabajo (2008). Certificación de competencias profesionales. Glosario de Términos Técnicos. Descargado el día 1 de julio de 2013. http:// www.oei.es/etp/certificacion_competencias_profesionales_glosario.pdf.

Perrenoud, P. (2007). Diez nuevas competencias para enseñar (4. ${ }^{a}$ ed.). Barcelona: Graó. 
Real Academia Española (2001). Diccionario de la Lengua española (22. ${ }^{a}$ ed.). Madrid: Espasa

Ríos Ariza, J. M. y Gómez Barajas, E. B. (2013). Relación entre competencias básicas de los estudiantes y competencias del profesorado. Revista Fuentes, 14, 209-230.

Sánchez Núñez, C.; Ramírez Fernandez, S. y García Guzmán, A. (2011). La formación de tutores externos de prácticum en educación: diseño, desarrollo y evaluación de una experiencia. Revista de Docencia Universitaria, vol. 9 (2), 119-145.

Tiana, A. (2011). Análisis de las competencias básicas como núcleo curricular de la educación obligatoria española. Bordón. Revista de Educación, 63 (I), 63-75.

Wisker, G.; Exley, K.; Antoniu, M. y Ridley, P. (2012). Trabajando individualmente con cada estudiante. Teoría personalizada, coaching, mentoría y supervisión en educación superior. Madrid: Narcea.

Zabala, A. y Arnau, L. (2008). 11 Ideas clave: cómo aprender y enseñar competencias. Barcelona: Graó, $4 .^{a}$ reimpresión.

Zabalza, M. A. (2001). Competencias personales y profesionales en el prácticum. Descargado el 21 de enero de 2014. http/www.redeberta.usc.es/uvi/public_html/images/pdf2001/ zabalza.pdf.

Zabalza, M. A. (2011). El prácticum en la formación universitaria: el estado de la cuestión. Revista de Educación, 354, 21-43. 